\title{
The effects of $\mu$ gradients on pulsations of rapidly rotating stars
}

\author{
Daniel R. Reese ${ }^{1}$, Francisco Espinosa Lara ${ }^{2}$ and Michel Rieutord ${ }^{3}$ \\ ${ }^{1}$ LESIA, CNRS UMR 8109, Observatoire de Paris, 92195 Meudon, France, \\ email: daniel.reese@obspm.fr \\ ${ }^{2}$ GEPI, CNRS UMR 8111, Observatoire de Paris, 92195 Meudon, France, \\ email: francisco.espinosa@obspm.fr \\ ${ }^{3}$ LATT, CNRS UMR 5572, Université de Toulouse, 31400 Toulouse, France \\ email: rieutord@ast.obs-mip.fr
}

\begin{abstract}
Recently, Reese et al. (2008), Lignières \& Georgeot (2008) and Lignières \& Georgeot (2009) showed that the frequencies of low-degree acoustic modes in rapidly rotating stars, also known as "island modes", follow an asymptotic formula, the coefficients of which can be deduced from ray dynamics. We investigate how this asymptotic behaviour is affected by $\mu$ gradients by comparing pulsation spectra from models with and without such a discontinuity.
\end{abstract}

Keywords. stars: oscillations, stars: rotation, stars: abundances

Rapid rotation strongly modifies both the geometry of pulsation modes and the organisation of the associated frequency spectrum. Recently, using both $2 \mathrm{D}$ calculations and ray dynamics, Reese et al. (2008), Lignières \& Georgeot (2008) and Lignières \& Georgeot (2009) showed that the frequencies of low-degree acoustic modes in rapidly rotating stars, also called "island modes", obey an asymptotic formula, for which two of the coefficients can be given in terms of travel time integrals. An open question is how such a formula will be affected by a strong compositional gradient.

In order to answer this question, we study the pulsation modes of rapidly rotating $3 M_{\odot}$ stellar models with the following compositions:

- Model 1: uniform composition $(X=0.70, Z=0.02)$

- Model 2: $\mathrm{H}$ poor interior $(X=0.35), \mathrm{H}$ rich envelope $(X=0.70)$

- Model 3: H rich interior $(X=0.70), \mathrm{H}$ poor envelope $(X=0.35)$

Model 3 is unrealistic, since nuclear reactions deplete hydrogen starting from the interior, but remains useful for the purposes of comparison. Furthermore, the density jumps in models 2 and 3 were chosen to be close to the surface in order to investigate the effects of $\mu$ gradients on acoustic modes. In reality, such jumps are likely to be located near the core and to have a stronger effect on gravito-inertial modes.

The models were produced using the ESTER code (Espinosa Lara \& Rieutord 2007, Rieutord \& Espinosa Lara 2009), which uses a multi-domain spectral approach, and a surface-fitting spheroidal coordinate system based on Bonazzola et al. (1998). In the present study, the rotation profiles have a constant value on the surface and were $r$ and $\theta$ dependant in the interior, as a result of baroclinic effects. The pulsation modes were calculated using the TOP code (Reese et al. 2009), fully including the effects of spheroidal distortion and differential rotation.

Figure 1 shows the meridional cross section of the same pulsation mode in models 2 and 3. The sound speed decreases across the jump when going towards the interior of model 2 , thereby causing the pulsation mode to be slightly deflected towards the centre, whereas the opposite occurs in model 3. 

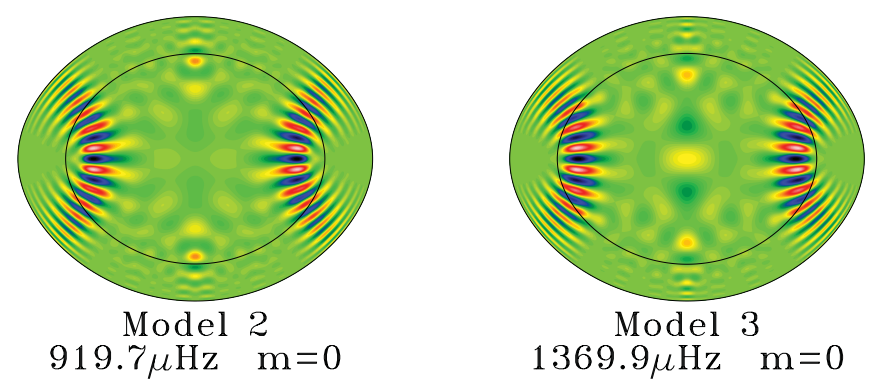

Figure 1. Meridional cross-section of the same pulsation mode in models 2 and 3 . The jump in sound speed, represented by the black internal line, causes a slight deflection of the mode.

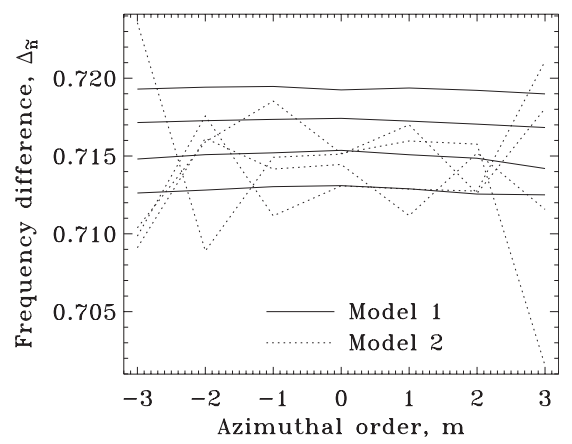

Figure 2. Frequency separations, as a function of $m$, between high order modes with $\tilde{\ell}=0$ and consecutive $\tilde{n}$ values.

Figure 2 shows the frequency separations, $\Delta_{\tilde{n}}=\omega_{\tilde{n}+1, \tilde{\ell}, m}-\omega_{\tilde{n}, \tilde{\ell}, m}$ of high order modes as a function of the azimuthal order, $m$. The pseudo-radial orders, $\tilde{n}$, go from 36 to 39. As expected, model 1 displays a smooth behaviour, since there are no $\mu$ gradients, whereas model 2 has an oscillatory behaviour. Nonetheless, these results must be taken with caution since the uncertainties on the frequencies of model 2, possibly as a result of numerical irregularities in the derivatives of $\Omega$, are of the same order of magnitude as the oscillations present in the figure.

\section{Acknowledgements}

DRR is currently supported by the CNES ("Centre National d'Etudes Spatiales") through a postdoctoral fellowship. The numerical calculations were carried out on the Vargas supercomputer (IBM SP Power 6) from the "Institut du Développement et des Ressources en Informatique Scientifique" (IDRIS). FEL thanks the ANR SIROCO.

\section{References}

Bonazzola, S., Gourgoulhon, E., \& Marck, J.-A. 1998, Phys. Rev. D, 58, 104020

Espinosa Lara, F. \& Rieutord, M. 2007, A\&A, 470, 1013

Lignières, F. \& Georgeot, B. 2008, Phys. Rev. E, 78, 016215

Lignières, F. \& Georgeot, B. 2009, $A \& \mathcal{E} A, 500,1173$

Reese, D., Lignières, F., \& Rieutord, M. 2008, A\&A, 481, 449

Reese, D. R., MacGregor, K. B., Jackson, S., Skumanich, A. et al. 2009, A\&A, 506, 189

Rieutord, M. \& Espinosa Lara, F. 2009, Communications in Asteroseismology, 158, 99 